\title{
Ansiedad precompetitiva en corredores de fondo en ruta en función de sus variables de entrenamiento Precompetitive anxiety in long-distance runners depending on their training variables \\ *Francisco Ruiz-Juan, **Antonio Zarauz Sancho, ***Gabriel Flores-Allende \\ *Universidad de Murcia (España), ${ }^{* *}$ IES Azcona (España), ***Universidad de Guadalajara (México)
}

\begin{abstract}
Resumen. En la práctica deportiva, estudiar las dimensiones de la ansiedad implicadas en que un sujeto desarrolle dicha actividad, se ha convertido en uno de los temas fundamentales de la psicología del deporte y del ejercicio. Se pretendía conocer qué relaciones predictivas presentaban sobre la ansiedad precompetitiva diversas variables de entrenamiento en una amplia muestra de 1795 corredores de fondo en ruta. De ellos, el $85.65 \%$ fueron hombres con una edad de $M=38.98$ ( $D T=10.45)$, y el $14.35 \%$ mujeres, con una edad $M=37.88$ ( $D T=9.80)$. La recogida de datos se llevó a cabo mediante la adaptación al castellano del Revised Competitive State Anxiety Inventory-2 (CSAI-2R) y de una serie de variables de entrenamiento. Se obtuvieron moderados valores de ansiedad cognitiva y somática y muy altos en autoconfianza, tanto en hombres y mujeres sin diferencias significativas por sexo, así como valiosos datos descriptivos de entrenamiento, que sirvieron para obtener unos sólidos modelos predictivos de la ansiedad y autoconfianza que se discuten.
\end{abstract}

Palabras clave. ansiedad; entrenamiento; autoconfianza; maratón.

Abstract. In sports, studying the dimensions of anxiety involved in an individual's practice of an activity has become one of the key themes of the psychology of sport and exercise. The study aim was to find out the predictive effects of various training variables on precompetitive anxiety in a large sample of 1795 long-distance runners. Of these, 85.65\% were men, aged $\mathrm{M}=38.98$ ( $\mathrm{SD}=10.45$ ), and $14.35 \%$ female, aged $\mathrm{M}=37.88$ (SD = 9.80). Data were collected using a Spanish adaptation of the Revised Competitive State Anxiety Inventory-2 (CSAI-2R) and several training variables. Moderate levels of cognitive and somatic anxiety and very high self-confidence were obtained, for both men and women, with no significant differences by gender, as well as valuable data describing training, which served to obtain solid predictive models of anxiety and self-confidence that were discussed. Key words. anxiety; training; self-confidence; marathon.

\section{Introducción}

Uno de los estados mentales característicos del deporte de competición es la ansiedad precompetitiva. Numerosos autores la han definido como una reacción emocional de aprehensión y tensión ante situaciones de competición (Dosil, 2004; Letelier, 2007; Martens, Vealy \& Burton, 1990) que, dependiendo del grado y forma en que se manifieste, condiciona en uno u otro sentido el rendimiento del deportista (Anshel, 1995; Gutiérrez, Estévez, García \& Pérez, 1997; Pozo, 2007; Smith, 1989). De hecho, puede llegarse a lo que Bueno, Capdevila y FernándezCastro (2002) llamaron sufrimiento competitivo, que se da cuando el deportista, durante el transcurso de su participación en una competición, alcanza la certeza de que no logrará en ella los objetivos que tenía previstos.

Por ello, la psicología del deporte ha estado siempre interesada en comprender mejor este constructo psicológico multidimensional. De hecho, ya Yerkes y Dodson (1908) explicaban en su conocido modelo de la U invertida, que el aumento del nivel de ansiedad va acompañado de una mejoría en el rendimiento, pero a partir de cierto nivel de ansiedad (activación moderada, que es la óptima) el rendimiento se deteriora.

Al diseñar y validar un instrumento para su medida, el Competitive State Anxiety Inventory-2 (CSAI-2), Martens et al. (1990) obtuvieron las tres dimensiones actualmente aceptadas de la ansiedad precompetitiva: ansiedad cognitiva, ansiedad somática y autoconfianza. La cognitiva evalúa las sensaciones negativas que el sujeto tiene sobre su rendimiento y las consecuencias del resultado. La somática hace referencia a la percepción de indicadores fisiológicos de la ansiedad, como tensión muscular, aumento de frecuencia cardíaca, sudoración y malestar en el estómago. La autoconfianza estima el grado de seguridad que el sujeto cree tener acerca de sus posibilidades de éxito en la competición.

En la actualidad, el instrumento de medida de referencia de estas tres dimensiones la ansiedad precompetitiva es el Competitive State Anxiety Inventory-2 Revised (CSAI-2R), de Cox, Martens y Russell (2003) que, al validarlo al español, Andrade, Lois y Arce (2007) lo redujeron a 16 ítems (véase apartado Instrumento). Los dos estados de ansiedad correlacionan significativa y positivamente entre sí, y significativa y negativamente con la autoconfianza (Buceta, López, Pérez-

Fecha recepción: 23-12-15. Fecha de aceptación: 30-04-16

Francisco Ruiz Juan

pacoruizjuan@gmail.com
Llantada, Vallejo \& Del Pino, 2003; Cervelló, Santos-Rosa, Jiménez, Nerea \& García, 2002). Aunque los hombres muestran mayor autoconfianza que las mujeres (Ruiz-Juan, Zarauz \& Flores-Allende, 2015a), son ellas quienes controlan mejor su ansiedad (Ponce de León, López, \& Medina, 2006). Sin embargo, en otros estudios fueron las mujeres las que mostraron mayores niveles de autoconfianza y menores niveles de ansiedad somática que los hombres (Arbinaga, 2013).

Pero no sólo el sexo del practicante es una variable que pueda influir significativamente en el grado en que se manifiestan los síntomas de la ansiedad, sino que otras muchas de índole personal o psicológico pueden hacer también que aumente la ansiedad y disminuya la autoconfianza. Así tenemos la menor edad del practicante (Arbinaga \& Caracuel, 2005; Zarauz \& Ruiz-Juan, 2013a), menor experiencia en competición (Mellalieu, Hanton \& O’Brien, 2004; Ruiz-Juan \& Zarauz, 2013), la percepción del deportista de no ir adecuadamente entrenado a competir (Gutiérrez et al., 1997), entrenar menos días a la semana (Zarauz \& Ruiz-Juan, 2013a), el menor nivel del deportista (Jones \& Swain, 1995; Pinto \& Vázquez, 2013), una mayor orientación al ego (Ruiz-Juan \& Zarauz, 2013; Zarauz \& Ruiz-Juan, 2013b), tener entrenador y bajo peso (Ruiz-Juan \& Zarauz, 2014) o, incluso, la percepción de la ansiedad como desfavorecedora del rendimiento (Pozo, 2007).

De la misma manera, hay otras variables de índole externa que no dependen del propio deportista y pueden influir en su ansiedad, como el ambiente en el que se encuentra compitiendo el deportista (Arbinaga \& Caracuel, 2005), el tipo y características de la prueba a la que se enfrenta (Jaenes, 2000), así como el tipo de deporte (Martens, et al., 1990; Pozo, 2007), el nivel de la competición (Zarauz \& Ruiz-Juan, 2013c), e incluso, si se es practicante de deporte individual o de equipo (Furst \& Tenembaum, 1984).

En la actualidad, existe una población de deportistas que no hace sino crecer exponencialmente cada año, la de corredores de fondo en ruta que participan habitualmente en pruebas de medio maratón y maratón. En ellos, Bueno et al. (2002) concluyeron que los corredores que mostraban mayor autoconfianza antes de la competición, tenían mayores probabilidades de alcanzar sus objetivos. Por el contrario, en los que mostraban mayor ansiedad cognitiva, esas probabilidades eran menores. Respecto a la ansiedad somática, concluyeron que no era demasiado importante, pues desaparecía en gran medida una vez iniciada la competición.

Queda clara pues la importancia del estudio de la ansiedad competitiva y sus dimensiones como facilitadoras del rendimiento deportivo. De la misma manera, como se ha demostrado en poblaciones similares 
(Zarauz \& Ruiz-Juan, 2013c), tanto los hábitos de entrenamiento como la experiencia de los deportistas, pueden actuar como moderadores de su ansiedad. Por ello, el objetivo de la presente investigación pasa por tratar de arrojar algo más de luz al respecto de qué variables de entrenamiento pueden ser predictoras de una mayor autoconfianza y una menor ansiedad precompetitiva en una amplia muestra de corredores de fondo en ruta y sus diferencias por sexo, dado que aún no están claras según la literatura consultada.

Se parte de la hipótesis de que tanto hombres como mujeres van a mostrar elevados niveles de autoconfianza y moderados en las dos dimensiones de la ansiedad, sin apenas diferencias significativas por sexo. Estas diferencias por sexo, sin embargo, si se esperan encontrar en las variables de entrenamiento que predicen la ansiedad y autoconfianza.

\section{Método}

\section{Participantes}

Se partió del total de corredores inscritos en los medios maratones de Almería y Elche (España) y Guadalajara (México) en 2010. Para asegurar que la muestra fuera representativa (error $\pm 3 \%$, intervalo de confianza 95.5\%), se empleó un diseño de muestreo estratificado por afijación proporcional teniendo en cuenta el sexo (86.65\% de hombres y 13.35\% de mujeres) y la edad. Se administró un cuestionario a 1060 corredores de fondo en ruta que participaron en los medios maratones deAlmería (30/01/2011), Elche(03/04/2011) y Guadalajara(20/02/11; 19/02/2012). Además, por medio de una página Web se obtuvieron las respuestas de 741 cuestionarios de corredores de maratón y medio maratón desde 01/03/11 a 20/03/13.

Por tanto, la muestra aleatoria y voluntaria fue de 1795 (1105 españoles, 690 mexicanos) corredores de fondo en ruta que estaba compuesta por 1541 hombres (85.7\%), con rango de edad de 18 a 76 años $(M=38.98$; $D T=10.45)$, y 254 mujeres (14.35\%), con rango de edad de 18 a 69 años $(M=37.88 ; D T=9.80)$. Así, se tuvo una muestra representativa con un error del $\pm 2.30 \%$ y un intervalo de confianza del $95.5 \%$

\section{Procedimiento}

En las carreras citadas anteriormente, se pidió permiso a la organización del evento mediante una carta en la que se explicaban los objetivos de la investigación, cómo se iba a realizar el estudio y se acompañaba un modelo del instrumento. El cuestionario fue administrado en un stand que se dispuso al efecto durante la recogida de dorsales de los atletas participantes el día previo a la carrera.

Para ampliar la muestra y obtener la más amplia y variada geográficamente en el territorio español, se solicitó colaboración al webmaster del principal foro de atletismo de España (http:// www.elatleta.com/foro/forum.php), al que se le pidió que colgara el cuestionario en la sección de corredores de ruta (http://www.retos.org/ encuesta/inicio.html).

En ambos casos, todos los sujetos fueron informados del objetivo del estudio, de la voluntariedad, absoluta confidencialidad de las respuestas y manejo de los datos, que no había respuestas correctas o incorrectas y se les solicitó que contestaran con la máxima sinceridad y honestidad. Además, se pidió que si habían contestado previamente este cuestionario no lo volvieran a hacer. Este trabajo posee el informe favorable de la Comisión de Bioética de la Universidad de Murcia.

Esta misma muestra y procedimiento ha sido empleada por los autores en otros estudios con diferentes objetivos, constructos psicológicos analizados, resultados y conclusiones (Ruiz-Juan \& Zarauz, 2014 Ruiz-Juan, Zarauz \& Flores-Allende, 2015b; Zarauz \& Ruiz-Juan, 2013a; Zarauz, Ruiz-Juan \& Flores-Allende, 2014).

\section{Instrumentos}

$>$ Inventario de Ansiedad Competitiva-2 Revisado; versión española de Andrade et al. (2007) de Revised Competitive State Anxiety Inventory-2 (CSAI-2R) de Cox et al. (2003). Tiene 3 subescalas: ansiedad cognitiva, ansiedad somática y autoconfianza. La primera y tercera contienen 5 ítems puntuables de 1 (nada) a 4 (mucho) con la que se obtiene una puntuación total entre 5 y 20 . La segunda contiene 6 ítems, que ofrece puntuaciones entre 6 y 24.

$>$ Hábitos de entrenamiento: promedios de kilómetros entrenados a la semana, tiempo por entrenamiento diario, días entrenados a la semana, porcentaje de tiempo que entrena solo a la semana, años corriendo, tener entrenador, compañeros de entrenamiento e índice de masa corporal.

\section{Análisis de los datos}

Correlación entre las subescalas (coeficiente de Pearson), diferencias de medias por sexo ( $t$ Student) y regresión lineal multivariante, se realizaron con SPSS 20.0.

\section{Resultados}

\section{Estadística descriptiva}

Tanto los hombres como las mujeres, que corren fondo en ruta presentaron valores moderados en ansiedad cognitiva y somática. Por el contrario, los valores en autoconfianza fueron muy elevados en ambas muestras, no existiendo diferencias estadísticamente significativas según el sexo (Tabla 1).

Las mujeres tenían entrenador en un porcentaje mayor (37.4\%) que los hombres (19.3\%), siendo estas diferencias significativas ( $\mathrm{p}<.001)$, al igual que dedicaban más minutos de entrenamiento en cada sesión, entrenaban más días a la semana y lo hacían con mayor número de compañeros que los hombres. Por el contrario, siempre con diferencias estadísticamente significativas, los hombres corrían más kilómetros semanales que las mujeres, entrenaban mayor porcentaje de tiempo solos, llevaban más años corriendo y tenían mayor IMC que las mujeres (Tabla 1).

Relaciones de la ansiedad precompetitiva con las variables de entrenamiento

La tabla 2 refleja las correlaciones calculadas. Las correlaciones entre la ansiedad cognitiva y la somática fueron moderadas y positivas, mientras que con la autoconfianza fueron bajas y negativas, en ambos sexos.

La ansiedad cognitiva correlacionó positivamente, en hombres, con kilómetros que corren a la semana, minutos de entrenamiento por sesión, días de entrenamiento por semana, y negativamente con IMC y tener entrenador. Sin embargo, en las mujeres, solo correlacionó negativamente el IMC.

En hombres, la ansiedad somática solamente correlacionó positivamente con kilómetros que corren a la semana y, negativamente, con años corriendo y tener entrenador. En mujeres las correlaciones no fueron significativas.

Las correlaciones en autoconfianza fueron muy similares tanto en hombres como en mujeres, de tal forma que correlacionó positivamente con minutos de entrenamiento por sesión, días de entrenamiento por semana y números de compañeros con los que entrena, y, negativamen-

\begin{tabular}{|c|c|c|c|c|c|c|}
\hline & $M_{\text {thamins }}$ & $D T$ & $M_{\text {sitions }}$ & $D T$ & t & $d$ \\
\hline Ansiedad cognitiva & 1.98 & 70 & 200 & .75 & .42 & -.02 \\
\hline Ansiedad somática & 2.22 & .74 & 2.25 & .76 & -66 & -.03 \\
\hline Autcoonfiarra & 3.29 & .61 & 3.27 & .70 & 39 & .03 \\
\hline Kmsemana & 51.17 & 25.78 & 43.89 & 25.64 & 4.17 & .28 \\
\hline Minutos entrenamiento por sesión & 78.47 & 29.49 & 82.62 & 32.96 & -2.03 & -.13 \\
\hline Dias enrenamiento por semana & 4.60 & 1.25 & 4.90 & 1.32 & -3.60 & -23 \\
\hline \%entrenamiento solo por semana & 67.76 & 34.01 & 56.88 & 37.73 & 4.64 & .30 \\
\hline Aîos carriendo & 9.22 & 8.84 & 8.20 & 7.83 & 1.72 & .12 \\
\hline$N^{N}$ compañeros entrenamiento & 292 & 5.71 & 5.04 & 9.57 & -4.89 & -26 \\
\hline IMC & 23.74 & 3.63 & 21.54 & 3.76 & ${ }^{12.23}$ & .59 \\
\hline \multicolumn{7}{|c|}{ 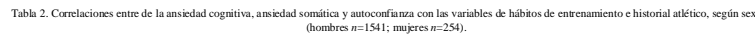 } \\
\hline & \multicolumn{2}{|c|}{ 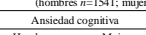 } & \multicolumn{2}{|c|}{$\begin{array}{l}\frac{\text { Ansieda somálica }}{\text { Hombres }} \\
\text { Mujeres }\end{array}$} & \multicolumn{2}{|c|}{ Aubconfianza } \\
\hline Ansiedad cognitiva & 1 & $\frac{1}{1}$ & $.59(*)$ & 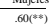 & 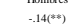 & 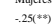 \\
\hline Ansiedad somática & $.59\left({ }^{*}\right)$ & $\left..600^{* *}\right)$ & 1 & 1 & $-1.10^{(* *)}$ & $-200^{(+\infty)}$ \\
\hline Autconffianza & $-.14\left(4^{* *}\right)$ & $-25(+4)$ & $.10^{(* *)}$ & $-20(+)^{(+)}$ & 1 & 1 \\
\hline $\begin{array}{l}\text { Kms semana } \\
\text { Minutos entrenamiento por sesín }\end{array}$ & . $\left.155^{(*)}\right)$ & .04 & $\left..06{ }^{*}\right)$ & .02 & .04 & .11 \\
\hline 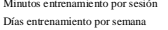 & 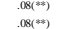 & . .07 & .04 & . .07 & . & $.17^{(2+*)}$ \\
\hline \%entrenamiento solo por semana & .03 & .02 & .00 & .04 & 年. & . \\
\hline Años carriendo & -.03 & -.05 & $-10\left(0^{*}\right)$ & -0.01 & .04 & \\
\hline$N^{N}$ compañeres en & .02 & .06 & .02 & -.00 & . $08^{(+\infty)}$ & . $14^{(\circ)}$ \\
\hline & $\left.-10^{* *}\right)$ & $-.14(7)$ & -.04 & -05 & .03 & .08 \\
\hline $\begin{array}{l}\text { Tener enrerendor } \\
\text { trox }\end{array}$ & $-.11\left(1^{(*)}\right.$ & .11 & $-0.07+(+\infty)$ & .04 & - $-10\left(0^{(*)}\right)$ & -.07 \\
\hline
\end{tabular}


te, con tener entrenador (sólo en hombres).

\section{Análisis regresivo multivariante}

Se realizó un análisis de regresión lineal multivariado, tratando de obtener unos modelos que explicasen la mayor parte posible de varianza. Se tomaron como variables dependientes las puntuaciones medias de la ansiedad precompetitiva (ansiedad cognitiva, ansiedad somática y autoconfianza). Las variables predictoras fueron cada una de las variables de entrenamiento: promedios de kilómetros entrenados a la semana, tiempo por entrenamiento diario, días entrenados a la semana, porcentaje de tiempo que entrena solo a la semana, años corriendo, tener entrenador, compañeros de entrenamiento e IMC. Como variable de selección se consideró el sexo.

Se extrajo el valor $\mathrm{R}^{2}$ para explicar la varianza, Beta para explicar la predicción entre variables, $\mathrm{F}$ para ver si existe relación entre variables seleccionadas y su significatividad (Tabla 3). Se obtuvieron modelos consistentes, ya que explicaron entre la mitad y casi los dos tercios de la varianza en hombres y mujeres.

El modelo de ansiedad cognitiva, en hombres, explica que se podía predecir significativamente por puntuar alto en ansiedad somática, kilómetros que entrena semanalmente, y por puntuar bajo en autoconfianza, IMC y tener entrenador (varianza: 61.7\%). En las mujeres, el modelo es bastante diferente a los hombres ya que la predicción fue por puntuar alto en ansiedad somática y días de entrenamiento a la semana, y por puntuar bajo en autoconfianza (varianza: 64.7\%).

El modelo de la ansiedad somática, en hombres, explica que se podía predecir significativamente por puntuar alto en ansiedad cognitiva y bajo en años que lleva corriendo (varianza: 60.1\%) y en las mujeres por puntuar alto en ansiedad cognitiva (varianza: 61.2\%).

La autoconfianza, en hombres, se predijo por puntuar alto en minutos de entrenamiento por sesión y días de entrenamiento a la semana, y por puntuar bajo en ansiedad cognitiva, kilómetros que entrena semanalmente y carecer de entrenador (varianza: 50.1\%). En las mujeres, el modelo fue bastante diferente a los hombres ya que la predicción fue por puntuar alto en días de entrenamiento a la semana y números de compañeros con los que entrena, y por puntuar bajo en ansiedad cognitiva (varianza: 57.2\%).

\begin{tabular}{|c|c|c|c|c|c|c|}
\hline & \multicolumn{2}{|c|}{ Ansiedad cognitiva } & \multicolumn{2}{|c|}{ Ansiedda somática } & \multicolumn{2}{|c|}{ Autoconfianza } \\
\hline & Hombes & Mujeres & Hombres & Mujeres & Hombres & Mujeres \\
\hline & Betatoso & Beter song & Betasish & Betatson & Betasing & Betatsine \\
\hline Ansiedda cognitiva & - & - & $.59+4$ & 6000+ & $-.14^{-\infty}$ & $-22^{2+*}$ \\
\hline Ansiedad somááca & $.57 \cdots$ & .55 +n- & -- & -. & -.02 & -.06 \\
\hline Autcoconfiarra & $-0.09+\cdots$ & $-.15^{\text {s+m}}$ & -01 & .04 & -- & -. \\
\hline Kmsemana & $.08+*$ & .02 & -.01 & .03 & $-0.8^{\circ}$ & .03 \\
\hline Minutos entrenanaiemto par sesión & .01 & -.05 & .01 & -.01 & . & .06 \\
\hline Días entrenamiento por semana & .01 & $.12^{\circ}$ & -01 & .03 & .14+世 & $.16^{*}$ \\
\hline \% entrenamiento solo por semana & .03 .03 & .04 & .03 & .05 & .03 & .01 \\
\hline Años corriendo & .01 & .03 & $-\infty 0^{-0 * \cdots}$ & .01 & .01 & -01 \\
\hline$N^{N}$ compañeros entrenamiento & -.02 & .04 & .01 & -.01 & .04 & $.12^{*}$ \\
\hline IMC & .0.5* & -.08 & .00 & .04 & -.02 & .07 \\
\hline Tener entrenador & .0.5* & .04 & -.01 & .01 & $-0.6^{\circ}$ & .02 \\
\hline
\end{tabular}

\section{Discusión}

La población de corredores de fondo en ruta de la presente investigación ha obtenido unos valores bastante moderados en ambos sexos de ansiedad cognitiva y somática, a pesar de ser un deporte individual y poder esperarse lo contrario (Furst \& Tenembaum, 1984). Ello podría deberse a múltiples factores. Quizá el principal sea que la inmensa mayoría de los corredores encuestados sean de carácter popular pues, por los datos de entrenamiento obtenidos, la mayoría de ellos ni cuenta con entrenador, ni entrena el número de días y kilómetros a la semana que los corredores de buen nivel. Por ello, su principal objetivo es simplemente terminar la prueba, lo cual modera significativamente su ansiedad frente a los corredores de mucho más nivel, cuyo mayor objetivo podría ser una marca que les permita acudir a una gran compe- tición o una beca, por ejemplo (Jones \& Swain, 1995; Pinto \& Vázquez, 2013).

De hecho, por las características de la prueba, la mayoría de participantes corren en pequeños grupos animándose mutuamente, lo que reduce nuevamente la presión que puedan sentir (Jaenes, 2000; LeónPrados, Fuentes \& Calvo, 2011; Pozo, 2007). Además, como explicaban Ruiz-Juan y Zarauz (2012), la mayoría de estos corredores tienen muchos años de experiencia entrenando y compitiendo en estas carreras, lo cual también modera la ansiedad que pudieran padecer(Mellalieu et al., 2004). Por otra parte, la mayoría de ellos son varones, adultos y con trabajo, lo cual son variables que pueden haber influido igualmente en la moderación de su ansiedad (Arbinaga \& Caracuel, 2005; Ponce de León et al., 2006; Ruiz-Juan \& Zarauz, 2014).

De la misma manera, hay otra serie de factores externos que pueden haber moderado la ansiedad precompetitiva en los participantes, como el ambiente previo al comienzo de la carrera, cuando todos los corredores pasan por la Feria del Corredor a recoger su dorsal y la bolsa del corredor, entablan conversación con otros competidores que hacía tiempo no veían, relajan tensiones, bromean..., de todo lo cual los autores de la presente investigación fueron testigos al administrar los cuestionarios.

Respecto a los altos valores obtenidos en ambos sexos en autoconfianza, además de por los motivos hasta ahora expuestos que han moderado significativamente ambas dimensiones de la ansiedad, podrían quedar explicados por los hábitos de entrenamiento obtenidos en esta población. El hecho de llevar bastantes años compitiendo y entrenando durante una media cercana a los cinco días por semana, como ya obtuvieran también Ruiz-Juan y Zarauz (2012), hace que se incremente su sensación de ir adecuadamente preparados a las competiciones, lo cual es sin duda motivo del aumento de su autoconfianza (Gutiérrez et al. 1997).

Que no haya habido diferencias significativas entre sexos en ninguna de las dimensiones de la ansiedad ni en la autoconfianza en la muestra de la presente investigación, contrasta con los resultados obtenidos recientemente en poblaciones similares de deportistas (Ruiz-Juan \& Zarauz, 2014; Zarauz \& Ruiz-Juan, 2013d), en donde los hombres mostraron mayores niveles de autoconfianza que las mujeres. La explicación al incremento de la autoconfianza en la muestra de mujeres de la presente investigación, muy probablemente pueda venir dada por los hábitos de entrenamiento de ellas, puesto que tenían entrenador en un porcentaje significativamente mayor que los hombres, dedicaban más minutos de entrenamiento en cada sesión, entrenaban más días a la semana y lo hacían con mayor número de compañeros que los hombres. Todo ello, sumado, les proporciona a ellas mayor sensación de ir adecuadamente preparadas a la hora de afrontar una carrera, incrementándoles por consiguiente su autoconfianza (Gutiérrez et al. 1997).

Por su parte, las correlaciones positivas de los dos dimensiones de la ansiedad entre si y negativas de ambas con la autoconfianza, no hacen sino confirmar todo lo hallado en estudios previos (Buceta et al., 2003; Cervelló et al., 2002; Ruiz-Juan \& Zarauz, 2014).

Además, de las coincidencias entre las correlaciones calculadas y los modelos predictivos de la ansiedad y autoconfianza en función de las variables de entrenamiento, podemos extraer algunas conclusiones. Por ejemplo, un bajo IMC correlacionó, tanto en hombres como en mujeres, con la ansiedad cognitiva, y la predijo en los hombres. Ello podría significar que aquellos corredores con bajo peso, al igual que sucediera en la investigación de Ruiz-Juan y Zarauz (2014), probablemente por el elevado número de días y kilómetros que entrenan, puedan percibir que se encuentran en condiciones de mejorar su mejor registro en la carrera y/o hacer un gran puesto acorde a su nivel, lo cual podría incrementarles su ansiedad cognitiva, máxime si no han sido dirigidos por un entrenador, como ocurre normalmente.

Otra variable de entrenamiento, el menor número de años corriendo, parece ser clave a la hora de incrementar la ansiedad somática de los varones, al igual que obtuvieran Mellalieu et al. (2004) y Ruiz-Juan y Zarauz (2013), pues correlaciona negativamente con ella y la predice. 
No obstante, como explicaban Bueno et al. (2002), esta dimensión de la ansiedad es menos preocupante, pues va disminuyendo significativamente conforme va avanzando la participación en la competición.

Otra variable, el mayor número de días a la semana de entrenamiento a la semana, tanto en hombres como en mujeres, ha resultado clave a la hora incrementar su autoconfianza, al igual que obtuvieran Zarauz y Ruiz-Juan (2013a). Sin duda, tanto por lo obtenido en las correlaciones como en los modelos predictivos de la presente investigación, el hecho de entrenar con más frecuencia, aun pudiéndoles incrementar su ansiedad somática, hace que aumente notablemente en todos los corredores su sensación de ir adecuadamente preparados para competir y, por consiguiente, su autoconfianza (Gutiérrez et al., 1997).

En los corredores varones, además, en esta sensación de ir adecuadamente preparado, influyó el mayor número de minutos que estos dedicaban por sesión. También en los varones, el hecho de no tener entrenador y hacer menos kilómetros a la semana, es otra de las variables que les incrementa su autoconfianza, mientras que en las mujeres es más decisivo un mayor número de compañeros de entrenamiento. Esto puede quedar explicado por la manera en la que estos corredores se toman su participación en la competición, siendo para la mayoría de ellos su único objetivo terminar la prueba, sea en el puesto que sea o con el tiempo que sea, como explicaban Jones y Swain (1995) y Pinto y Vázquez (2013), por eso se sienten más seguros compitiendo a un ritmo de carrera controlado, junto con los compañeros de entrenamiento que les animan continuamente, sobre todo las mujeres.

Para investigaciones futuras, podría ser interesante contrastar los hallazgos de la presente investigación en corredores con diferentes niveles; popular, medio y élite.

\section{Referencias}

Andrade, E. M., Lois, G., \& Arce, C. (2007). Propiedades psicométricas de la versión española del Inventario de Ansiedad Competitiva CSAI-2R en deportistas. Psicothema, 19(1), 150-155.

Anshel, M. (1995). Anxiety. En T. Morris y J. Summers (eds.): Sport Psychology: Theory, applications \& issues (pp. 29-62). Brisbane, CA: John Wiley \& Sons.

Arbinaga, F. (2013). Fisicoculturismo: diferencias de sexo en el estado de ánimo y la ansiedad competitiva. Revista de Psicología del Deporte, 22(2), 353-360.

Arbinaga, F., \& Caracuel, J.C. (2005). Precompetición y ansiedad en fisicoculturistas. Revista de Psicología del Deporte, 14(2), 195208

Buceta, J., López, A., Pérez- Llantada, M., Vallejo, M., \& Del Pino, M. (2003). Estado psicológico de los corredores populares de maratón en los días anteriores a la prueba. Psicothema, 15, 273-277.

Bueno, J., Capdevila, L., \& Fernández-Castro, J. (2002). Sufrimiento competitivo y rendimiento en deportes de resistencia. Revista de Psicología del Deporte, 11(2), 209-226.

Cervelló, E., Santos-Rosa, F., Jiménez, R., Nerea, A., \& García, T. (2002). Motivación y ansiedad en jugadores de tenis. Motricidad, 9, 141-161.

Cox, R., Martens, M., \& Russell W. (2003). Measuring anxiety in athletics: The revised Competitive State Anxiety Inventory-2. Journal of Sport and Exercise Psychology, 25, 519-533.

Dosil, J. (2004). Psicología de la actividad física y del deporte. Madrid: McGraw Hill.

Furst, D.M., \& Tenembaum, G (1984). Sportperformance and various state anxiety components: Across sectional study. Netanya:Wingate Institute.

Gutiérrez, M., Estévez, A., García, J. \& Pérez, H. (1997). Ansiedad y rendimiento atlético en condiciones de estrés: efectos moduladores de la práctica. Revista de Psicología del Deporte, 6(2), 27-46.

Jones, G., \& Swain,A. (1995). Predisposition to experience debilitative and facilitative anxiety in elite and non-elite performers. The Sport Psychologist, 9, 201-211.
Letelier, A. (2007). Estudio correlacional entre la Ansiedad Estado Competitivay las Estrategias de Afrontamiento Deportivo en tenistas juveniles. Tesis de Licenciatura. Universidad de Chile.

León-Prados, J., Fuentes, I. \& Calvo, A. (2011). Ansiedad estado y autoconfianza precompetitiva en gimnastas. Revista Internacional de Ciencias del Deporte, 23, 76-91.

Martens, R., Vealey, R.S., \& Burton, D. (1990). Competitive anxiety in sport. Champaign, IL: Human Kinetics.

Mellalieu, S., Hanton, S., \& O’Brien, M. (2004). Intensity and direction of competitive anxiety as a function of sport type and experience. Scandinavian Journal of Medicine and Science in Sports, 14, 326334.

Pinto, M.F., \& Vázquez, N. (2013). Ansiedad estado competitiva y estrategias de afrontamiento: su relación con el rendimiento en una muestra argentina de jugadores amateurs de golf. Revista de Psicología del deporte, 22(1), 47-52.

Ponce de León, Y., López, J., \& Medina, M. (2006). Habilidades psicológicas en los atletas de primera fuerza en atletismo. Revista de Ciencias del Ejercicio -FOD-, 2, $42-57$.

Pozo, A. (2007). Intensidad y dirección de la ansiedad competitiva y expectativas de resultado en atletas y nadadores. Revista de Psicología del Deporte, 16(2), 137-150.

Ruiz-Juan, F. \& Zarauz, A. (2012). Variables que hacen adicto negativamente a correr al maratoniano español. Retos, 21, 38-42.

Ruiz-Juan, F. \& Zarauz, A. (2013). Análisis de la ansiedad en el atletismo; un estudio con veteranos. Revista Internacional de Ciencias del Deporte, 33(9), 222-235. Doi; 10.5232/ricyde2013.03302

Ruiz-Juan, F. \& Zarauz, A. (2014). Ansiedad en maratonianos en función de variables socio-demográficas. Retos, 25, 28-31.

Ruiz-Juan, F., Zarauz, A., \& Flores-Allende (2015a). Variables predictoras de la ansiedad precompetitiva: aspectos diferenciales en corredores de fondo en ruta. Universitas Psychologica, 14(3), 1047-1058.

Ruiz-Juan, F., Zarauz, A., \& Flores-Allende (2015b).Percepción del éxito en corredores de fondo en ruta en función de variables sociodemográficas. Retos, 27, 136-139.

Smith, R. (1989). Athletic stress and burnout: conceptual models and intervention strategies. En D. Hackfort \& C.D. Spielberger (eds.): Anxiety in sports: An international perspective (pp. 183-201). Nueva York, NY: Hemisphere.

Yerkes, R.M., \& Dodson, J.D. (1908). The relation of strength of stimulus to rapidity of habit-formation. Journal of Comparative Neurology and Psychology, 18, 459-482.

Zarauz, A., \& Ruiz-Juan, F. (2013a). Motivaciones de los maratonianos según variables socio-demográficas y de entrenamiento. Retos, 24, 50-56.

Zarauz, A., \& Ruiz-Juan, F. (2013b). Ansiedad, satisfacción, percepción y creencias sobre las causas del éxito en atletas veteranos españoles. Ansiedad y Estrés, 19(1), 83-93.

Zarauz, A., \& Ruiz-Juan, F. (2013c). Variables predictoras de la ansiedad en atletas veteranos españoles. Retos, 23, 29-32.

Zarauz, A. \& Ruiz-Juan, F. (2013d). Análisis de la ansiedad en el atletismo; un estudio con veteranos. Revista Internacional de Ciencias del Deporte, 9(33), 225-235.

Zarauz, A., Ruiz-Juan, F., \& Flores-Allende (2014). Compromiso con el entrenamiento y competición de los maratonianos según variables socio-demográficas. Retos, 26, 118-121.

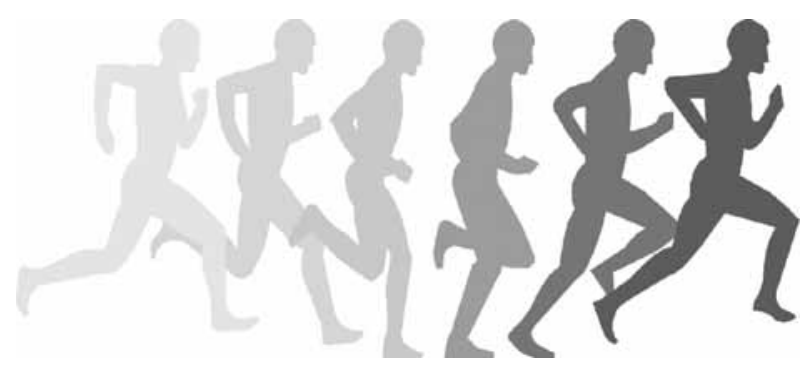

